without affecting recency effects, a result consistent with the assumption (Glanzer and Cunitz, 1966) that different storage mechanisms are involved in primacy and recency. Finally, the figure shows recency effects to be somewhat more pronounced than primacy effects, a finding consistent with prior FR studies (e.g., Murdock, 1962).

The failure to obtain the Type of Instructions $\times$ Serial Position and Type of Instructions $\times$ Presentation Rate $\times$ Serial Position interactions raises doubts as to the validity of the differential-rehearsal hypothesis. The occurrence of both interactions depended upon $S$ 's ability to follow NR instructions. Supporting the hypothesis was the significant Presentation Rate $\times$ Serial Position interaction, the occurrence of the interaction not being dependent upon $S$ 's following NR instructions.

\section{REFERENCES}

Glanzer, M., and Cunitz, A. R. Two storage mechanisms in free recall. J. verb. Learn. verb. Behav., 1966, 5, 351-360.

MURDock, B. B., JR. The serial position effect of free recall. J. exp. Psychol., 1962, 64, 482-484.

THORNDIKE, E. L., AND LORGE, I. The teacher's word book of 30,000 words. New York: Bureau of Publications, Teachers College, Columbia University, 1944.

Waugh, N. C., and Norman, D. A. Primary memory. Psychol. Rev., 1965, 72, 89-104.

(Received February 5, 1968)

\title{
Distraction and Originality in Word Associations
}

\author{
JOHN C. MASTERS ${ }^{\mathfrak{l}}$ \\ University of Minnesota, Minneapolis, Minnesota 55455 \\ AND \\ GARY B. Mesibov \\ University of Michigan, Ann Arbor, Michigan 48108
}

\begin{abstract}
Two types of auditory distraction were employed during a word-association task: A distractor to be ignored (inhibition distractor) and a distractor to be attended to (vigilance distractor). A control group received no distraction. Houston and Jones (1967) presented evidence that the presence of an inhibition distractor improved performance on a task postulated to involve inhibition. If originality in word associations involves the inhibition of more probable common responses, any manipulation (inhibition distractor) which increases inhibition facility should increase originality and/or decrease the latency of original responses. This study found no such effect with respect to the originality of responses. Latency of response yielded a marginal effect in the predicted direction. The results are discussed in terms of the similarity of experimental tasks and measures between this study and the one by Houston and Jones.
\end{abstract}

Houston and Jones (1967) hypothesized that performance on a task which required the inhibition of a prominent response (The Stroop Color-Word Test) could be improved if $S$ was subjected to a variety of distracting noises which he was told to ignore. Their prediction was confirmed. Subjects who were told to ignore an auditory distractor performed better on such

1 This project was carried out while the senior author held NIH postdoctoral fellowship 1-F2-MH-23, 461-01 (PS) at the Laboratory of Human Development, Stanford University. a task than did $S$ s who were instructed to listen for, and respond to, a cue embedded in the same auditory distractor. Confirmation of this hypothesis may be interpreted to increase the construct validity of the hypothesis that inhibitory processes interact. The performance of one task requiring inhibition (ignoring a distractor) may enhance the performance of a second, simultaneous task which also requires inhibition.

Masters and Anderson (1968) report that the originality of word associations may be increased by either instructing the $S$ to "be more original" or by 
increasing the length of time following the presentation of the stimulus word in which $S$ may produce his association. These effects appear additive. The results were interpreted in terms of a response-hierarchy model (Mednick, 1962). In order to produce an original word association, $S$ must actively inhibit his first response choice in order to emit a less common response which is lower in the hierarchy of responses to a particular stimulus word.

The present study attempted a synthesis of the two above studies. It was hypothesized that the presence of a distractor would differentially affect the originality of $S$ s depending upon whether they were: (a) required to ignore the distractor (Inhibition Distractor) or (b) required to attend to the distractor (Vigilance Distractor). It was predicted that the presence of an inhibition distractor would increase the originality of word associations, and that the presence of a vigilance distractor would have no effect or would be detrimental to the production of original word associations. It was also anticipated that the facilitating effect of an inhibition distractor might be observable in $S$ 's response latencies such that they would be able to produce original word responses more quickly than $S$ s experiencing a vigilance distractor.

Procedure. Forty-eight college students were randomly assigned to one of three groups: Inhibition Distraction (ID), Vigilance Distraction (VD), and Control (C). The taped auditory distractor was identical to the one employed by Houston and Jones (1967). It was comprised of "A variety of familiar sounds, e.g., trains, dripping water, etc, and unfamiliar sounds, e.g., electronic music, gibberish, etc." All groups associated to one list of words (List 1) without instructions other than to provide relevant associations. All groups were then instructed to be as original as possible, donned earphones and associated to a second list of words (List 2). In ID, Ss were instructed to ignore completely the distractor tape. In VD, they were told to tap the table with their pencil whenever they heard the spoken word "tap" which was embedded in the distracting noises. In $\mathrm{C}, S \mathrm{~s}$ performed the task without auditory distraction though still wearing the earphones.

Stimulus words were arranged in two lists provided by Maltzman (1958), Lists A and B. The order of presentation of the two lists was counterbalanced across all $S$ s. The latency of $S$ 's response to each stimulus word was recorded by $E$.

Originality scores were derived from the data. On the second list of words to which $S$ associated, the originality score for a response to stimulus word " $N$ " on List $\mathbf{A}$ was simply the number of persons who had given that response when taking List $A$ under the instruction merely to give relevant associations. For purposes of clarity in discussion, ${ }^{-}$this score was subtracted from a constant so that a high score indicates high originality.

Results and Discussion. A summary of the data appears in Table 1. The three groups were comparable in their mean originality scores for List 1. Analysis of variance of the originality scores for List 2 revealed no differences among the groups, $F(2,45)=1.56$. If Ss selected original responses by sorting through their hierarchy of responses to each stimulus, original responses should have shown greater latency. Given that there was no difference in the originality of response the effect of any interaction of inhibition processes might have been to lower the length of time necessary to achieve the selection of acceptable responses. Mean latencies for the three groups were comparable on List 1. Since the distribution of latencies on List 2 was skewed, the analysis employed was the Kruskal-Wallis one-way analysis of variance. Results indicated a nonsignificant tendency for the ID group to have a lower latency than the other two groups, $H(z)=4.58, .10>$ $p>.05$.

It is possible that the results for originality scores were constricted by a ceiling effect. The maximum originality possible in the present scoring system was 24.0 , and it is apparent that the means approached this value. Responses were also scored in terms of the Palermo-Jenkins word-association norms (Palermo and Jenkins, 1964). For this analysis, a word was given a commonality score which was the number of college students from the 500 of each sex assessed who gave a given response to a particular stimulus. These commonality scores were subtracted from a constant so that a high score indicates high originality. This, core

TABLE 1

Summary OF THE DATA ${ }^{a}$

\begin{tabular}{llrl}
\hline & ID & VD & C \\
\hline $\begin{array}{l}\text { Latency, List 1 } \\
\quad \text { (sec) }\end{array}$ & 2.06 & 2.05 & 2.08 \\
$\begin{array}{l}\text { Latency, List 2 } \\
\quad(\mathrm{sec})\end{array}$ & 3.82 & 5.68 & 5.29 \\
$\begin{array}{l}\text { Originality, List 1 } \\
\quad \text { Max = 24.0) }\end{array}$ & 15.70 & 16.45 & 15.27 \\
$\begin{array}{l}\text { Originality, List 2 } \\
\quad \text { (Max = 24.0) }\end{array}$ & 22.02 & 23.02 & 22.52 \\
$\begin{array}{l}\text { Palermo-Jenkins, List 1 } \\
\quad \text { (Max = 150) } \\
\text { Palermo-Jenkins, List 2 }\end{array}$ & 19.31 & 35.28 & 7.19 \\
$\quad$ (Max = 150) & 120.94 & 137.28 & 125.07 \\
\hline
\end{tabular}

'Note: An individual's score is the mean latency, originality, or commonality for all his responses to a particular list. The above scores are the group means, each based on 16 cases. 
appeared to be measuring originality in a way similar to the above described originality scores since the correlations between the two methods of scoring were +.93 for List 1 and +.93 for List 2 . It appears from Table 1, however, that the Palermo-Jenkins scores were not so close to a ceiling as were the originality scores. Since the three groups did not have equivalent originality scores on List 1 , analysis of variance was carried out on change scores, scorc on List 1 minus score on List 2. Again there were no differences among the groups, $F<1$.

Thus the hypothesis that inhibition processes may interact was not confirmed for the present experimental task and materials. The word-association task employed in this experiment may be characterized as involving selection and retrieval of words from a storage hierarchy, while the Houston and Jones task (the Stroop) appears to involve interference effects from competing but not necessarily hierarchically arranged response tendencies. It is interesting to note that the marginal significance obtained in the present study is for a timemeasure, and the measures employed in the Houston and Jones study were also time-measures reflecting the speed of response.

It has been suggested ${ }^{2}$ that the constituent noises in the distraction tape employed, which was the same as used in the Houston and Jones study, were too varied

${ }^{2}$ B. Kent Houston, personal communication. in nature and thus could not easily and consistently be ignored. It is not clear, however, why this factor should differentially affect the Stroop and word-association tasks. A replication of the Houston and Jones experiment would be wise. Such a study, as well as a replication of the present study, might well utilize a less complex distractor whose components are less varied than those in the current distractor but sufficiently complex to prevent simple habituation.

\section{REFERENCES}

Houston, B. K. AND Jones, T. M. Distraction and Stroop Color-Word Performance.J.exp.Psychol., $1967,74,54-56$.

Maltzman, I., Bogartz, W., and Breger, C. A Procedure for Increasing Word Association Originality and Its Transfer Effects. $J$. exp. Psychol., 1958, 56, 392-398.

Masters, J. C., And Anderson, G. W. Time and Delay as Determinants of Originality in Word Associations. J. verb. Learn. verb. Behav., 1968, 7, 432433.

Mednick, S. A. The Associative Basis of the Creative Process. Psychol. Review, 1962, 69, 220-232.

Palermo, D. S., AND Jenkins, J. J. Word Association Norms: Grade School Through College. Minneapolis: University of Minnesota Press, 1964.

(Received February 28, 1968)

\title{
Comparisons of Individual and Cultural Response Hierarchies Based on Continued Association ${ }^{1}$
}

\author{
Gail A. BRUder \\ State University of New York at Buffalo, New York 14214
}

\begin{abstract}
Individual response protocols of eight associations to each of eight stimulus words (Buffalo sample) were compared with cultural response hierarchies (Pittsburgh sample). The number of individual responses which matched responses in the top eight hierarchy positions (HPs) of the norms was related to the rank of those responses in the norms. Cultural hierarchies, derived in different ways, were similar and did not differ in degree of individual-cultural response agreement. It was concluded that prediction of individual responses, but not their HPs, is possible on the basis of the top ranking responses from norms.
\end{abstract}

It has often been assumed that hierarchies of responses based on cultural frequency refiect the response hierarchies of individuals. Two studies have compared

1 This research was supported in part by funds from the Biomedical Sciences Support Grant (NIH) administered by the Research Foundation of State University of New York. cultural response hierarchies, derived from the Minnesota norms (Russell and Jenkins, 1954), with individual hierarchies based on order of emission in continued association. Rosen and Russell (1957) found agreement between cultural frequency and the order of emission when two successive responses were obtained. Osipow and Grooms (1965) compared the response ranks in cultural and individual continued association 